\title{
Taste aversion learning and schedule-induced polydipsia in rats
}

\author{
NIGEL W. BOND \\ School of Behavioral Sciences, Macquarie University, North Ryde, N.S.W. 2113, Australia \\ and \\ P. KEITH CORFIELD-SUMNER \\ University of Birmingham, Birmingham B15 2TT, England
}

\begin{abstract}
Experiment 1 sought to determine whether schedule-induced drinking could be abolished by means of a taste aversion. Polydipsic rats were given access to a $.4 \%$ saccharin solution while they were exposed to an intermittent food schedule. Immediately after the session, they received an intraperitoneal injection of either lithium chloride or sodium chloride. Following a recovery day with water in the experimental chamber, the animals were again exposed to the saccharin solution. The poisoned animals (lithium chloride) drank very little saccharin compared to the control animals (sodium chloride), indicating that they had learned a taste aversion in oniy one conditioning trial. Experiment 2 established that polydipsic rats can learn a taste aversion despite a long delay between schedule-induced saccharin consumption and poisoning, and that the delay gradient displayed by polydipsic rats is similar to that observed in thirst-motivated rats.
\end{abstract}

Food-deprived rats exposed to an intermittent schedule of food reinforcement will consume an excessive amount of water if it is made available during the experimental session, even though they are never water deprived. For example, Falk (1961) reported that rats' water intake during a 3.17-h session may be three to four times their normal daily intake, and he termed this phenomenon "schedule-induced polydipsia."

A number of studies have demonstrated that schedule-induced polydipsia has motivating properties. Falk (1966) trained food-deprived rats to barpress for food on a variable-interval 1-min schedule of reinforcement. Water was freely available in the home cage, but in the experimental chamber the delivery of $.1 \mathrm{ml}$ of water was made contingent upon pressing another bar. Falk found that rats maintained a polydipsic level of drinking even when water was available only on a fixed-ratio 50 reinforcement schedule. Since the rats were not water-deprived, barpressing for water in the experimental chamber was clearly due to the strong motivation to drink produced by the intermittent food schedule.

Utilizing a taste aversion paradigm, Roll, Schaeffer, and Smith (1969) presented a saccharin solution to polydipsic rats. After drinking the saccharin, the rats were exposed to ionizing radiation. Although this pro-

This research was carried out while Nigel Bond was on study-leave at Birmingham University. The authors wish to thank Professor P. L. Broadhurst for the use of valuable facilities and for his encouragement and advice throughout this period. Reprints may be obtained from Nigel Bond. cedure produces a marked aversion to saccharin in thirsty rats following only a single experience, repeated pairings of the saccharin solution and ionizing radiation were necessary to reduce the high levels of drinking generated by the intermittent food schedule. Again, these results appear to show that schedule-induced polydipsia is a highly motivated activity. However, in contrast to the Roll et al. findings, Bond, Blackman, and Scruton (1973) found that the licking associated with schedule-induced polydipsia was relatively more sensitive to a variety of suppression procedures than was operant barpressing. When exposed to punishment by response-contingent electric shock, complete suppression of licking occurred with no recovery, whereas when barpressing was punished partial recovery was evidenced. Further, when exposed to a conditionedsuppression procedure, there was more suppression of licking than of barpressing, even though conditions were identical.

One problem with the Roll et al. study is that it made no direct comparison between schedule-induced and thirst-motivated drinking. If their relative failure to produce a taste aversion in polydipsic animals is to be regarded as a unique property of schedule-induction, a thirst-motivated group is necessary to establish that the conditions employed would produce a taste aversion in thirsty animals. The present experiment therefore looked at the effects of pairing schedule-induced saccharin consumption with an injection of lithium chloride ( $\mathrm{LiCl}$ ), a drug effective in producing taste aversions in thirst-motivated rats (cf. Revusky \& Garcia, 1970). As a comparison, a group of thirst- 
motivated animals was also run to establish that the conditions employed would lead to a taste aversion in thirsty animals. To keep conditions constant, the thirstmotivated animals were food deprived and received the same number of food pellets in the experimental chamber as the polydipsic rats, but not on an intermittent schedule. Further, the experimental sessions were of such a duration as to insure similar fluid intakes in the polydipsic and thirst-motivated animals (cf. Bond \& Di Guisto, 1975).

\section{EXPERIMENT 1}

\section{Method}

Subjects. Eighteen male hooded rats of a laboratory-bred strain served as subjects. They were 150 days old and were maintained at $85 \%$ of their preexperimental weights, which ranged from 365 to $431 \mathrm{~g}$. Each animal was individually housed, and the 12 polydipsic animals had water freely available at all times in their home cages. The remaining six animals formed the thirstmotivated group and had access only to fluid in the experimental chambers.

Apparatus. The apparatus consisted of six standard two-lever operant test chambers (Lehigh Valley Electronics) housed in sound-and lightproof outer cubicles. The levers were inoperative throughout the experiment. Food in the form of $45-\mathrm{mg}$ pellets of standard rat food (P. J. Noyes Co.) could be dispensed to the animals through chutes positioned in the center-bottom of the front walls. In each chamber the stimulus light, situated to the right of and $8 \mathrm{~cm}$ higher than the food chute, was removed and a water bottle was positioned behind the front panel so that the spout hung approximately $5 \mathrm{~mm}$ behind the resulting hole. The rats could easily reach through the hole to lick the spout. Programming of the experiments was achieved by means of an on-line digital computer (PDP-9, Digital Equipment Corporation) programmed in ACT-9, a language designed for the control of psychological experiments (Millenson, 1971).

Procedure. The rats were given two $1-h$ sessions in the chambers to allow them to adapt to the novel environment. During these sessions, a few food pellets were placed in the food chutes and the water bottles were in position.

For the next 20 consecutive daily sessions, the 12 animals in the polydipsic group were exposed to an intermittent food schedule in which 31 food pellets were dispensed individually at 1-min intervals. As already noted, the animals in this group had water freely available in the home cages. The six animals in the thirstmotivated group were placed in the chambers for $30 \mathrm{~min}$ daily. This period was the only time they were allowed access to water. They received 31 food pellets placed in a dish at the beginning of the session. Fluid intakes were recorded to the nearest milliliter.

On Day 21 (conditioning day), the drinking fluid was changed from water to a $.4 \%$ saccharin solution. Immediately following the end of the experimental session, six of the polydipsic animals (SIP-E) and the six thirst-motivated animals each received an intraperitoneal injection of $10 \mathrm{ml} / \mathrm{kg}$ of $.3 \mathrm{M}$ lithium chloride (LiCl). The remaining polydipsic animals (SIP-C) received an intraperitoneal injection of $10 \mathrm{mi} / \mathrm{kg}$ of $.3 \mathrm{M}$ sodium chloride $(\mathrm{NaCl})$. All animals were then returned to their home cages, and water was removed for a period of $6 \mathrm{~h}$. At the end of this time, all the animals were given their daily food ration and placed on free water.

On Day 22, all animals received their daily food ration in their home cages and the thirst-motivated animals were allowed access to water for $30 \mathrm{~min}$ while they consumed their food.

Day 23 served as a recovery day. All animals were placed in the experimental chambers with water as the available fluid and the
31 food pellets available either at the beginning of the session (thirst-motivated group) or dispensed individually at 1-min intervals (polydipsic groups).

On Day 24 , the animals were tested for their aversion by being placed in the experimental chambers with the intermittent food schedule in operation and with the $.4 \%$ saccharin solution as the available drinking fluid.

Data analysis. Between-groups analyses were carried out using the Mann-Whitney $U$ test, and within-groups analyses employed the Wilcoxon T test. All comparisons were two-tailed.

\section{Results}

Mean fluid intakes in the experimental chambers for each of the three groups are shown in Figure 1. There were no significant differences in water consumption between any of the groups on the last baseline day (B). During this baseline phase, all the animals exposed to the intermittent food schedule (Groups SIP-E and SIP-C) developed a pattern of behavior which consisted of taking each pellet as it was delivered and then going immediately to the water spout and drinking. Further, taking into account the session length, they exhibited levels of water intake comparable to those reported previously for this strain (cf. Cope, Sanger, \& Blackman, 1976). Therefore, given the patterning of their

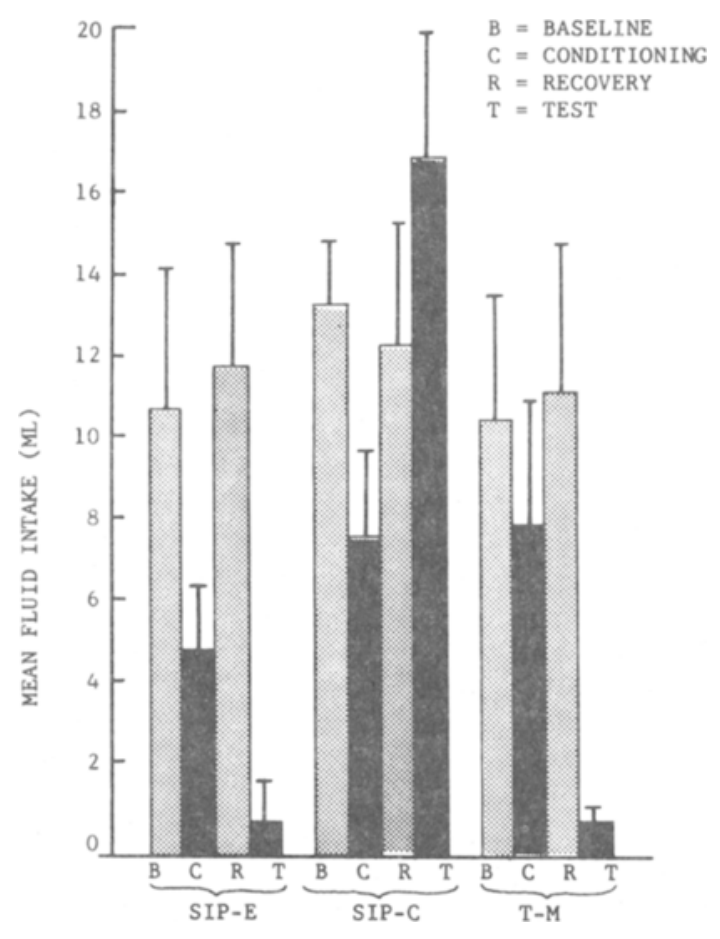

Figure 1. Mean fluid intakes in the experimental chambers of the poisoned polydipsic animals (SIP-E), polydipsic controls (SIP.C) and thirst-motivated animals (T-M) on Day 20, the last baseline day $(B)$, conditioning day $(C)$, recovery day $(R)$, and test day $(T)$. Standard deviations are shown by the vertical lines. The light hatching indicates that water was available in the experimental chambers; the dark hatching indicates that the saccharin solution was available. 
drinking and the amounts they consumed, one can conclude that the animals exposed to the intermittent food schedule (Groups SIP-E and SIP-C) displayed schedule-induced polydipsia.

When saccharin solution was made available on the conditioning day $(\mathrm{C})$, all three groups showed a significant decrease in fluid consumption compared to baseline ( $\mathrm{T}=0, \mathrm{p}<.05$, in all cases), but there were no significant differences between the groups. In the polydipsic animals (Groups SIP-E and SIP-C), this decline in fluid consumption was evidenced by a reduction in the number of food pellets followed by drinking. However, in those intervals where drinking occurred it took the same form as described in the previous paragraph. Further, while the amounts consumed may not seem excessive, note that the polydipsic groups (SIP-E and SIP-C), which had water available in their home cages, ${ }^{1}$ drank as much saccharin solution in the experimental chambers as the thirst-motivated group, which had no other source of fluid (cf. Figure 1).

On the recovery day $(\mathrm{R})$, all three groups recovered their baseline levels (B) of water-intake.

Saccharin was reintroduced into the experimental chamber on the test day (T). The SIP-E and thirstmotivated groups both showed significant declines in saccharin consumption compared to the conditioning day $(\mathrm{C})(\mathrm{T}=0, \mathrm{p}<.05$ in both cases), but did not differ from each other $(U=10.5, p>.05)$. In contrast, Group SIP-C evidenced a significant elevation in saccharin consumption compared to the conditioning day $(\mathrm{C})(\mathrm{T}=0, \mathrm{p}<.05)$, and animals in this group consumed significantly more saccharin on the test day (T) than Group SIP-E $(U=0$, $p<.002)$. These two groups differed only in that Group SIP-E received an injection of $\mathrm{LiCl}$ immediately after tasting saccharin, whereas Group SIP-C received an injection of $\mathrm{NaCl}$.

This pattern of results suggests that a taste aversion can be conditioned in polydipsic animals following only one pairing of the novel taste with illness (Group SIP-E). Further, under conditions where polydipsic (SIP-E) and thirst-motivated groups were kept as alike as possible, both displayed similarly strong aversions.

\section{Discussion}

The present findings provide a clear demonstration that schedule-induced polydipsia is amenable to suppression in a typical taste aversion paradigm. It required only one pairing of the novel taste (saccharin) and illness (injection with lithium chloride) to markedly reduce subsequent saccharin consumption. Further, the technique was as effective in suppressing schedule-induced drinking as it was in suppressing thirst-motivated drinking. The question then arises as to how these results can be reconciled with those of Roll et al. (1969), who reported little change in saccharin consumption following a single pairing of the saccharin solution with ionizing radiation. A number of problems related to their study have already been outlined, but one important point may be added. The session length in the Roll et al. study was $2 \frac{1}{2} \mathrm{~h}$. This meant that the animals tasted saccharin at least $2 \frac{1}{2} \mathrm{~h}$ before being exposed to irradiation, and thus for an even longer period before the onset of illness. The actual time between the initial experience of a novel taste and the onset of illness has been shown to be an important determinant of the strength of the subsequent aversion, since animals learn that the taste is "safe" during this period (Kalat \& Rozin, 1973). In our own study, session length was $30 \mathrm{~min}$; thus, animals were injected soon after their initial taste of saccharin. As such, the different degrees of aversion obtained in the two studies may be a simple reflection of the difference in time from the initial experience of the novel taste to the onset of illness.

\section{EXPERIMENT 2}

In the first experiment, it was established that schedule-induced saccharin consumption could be abolished following only one pairing of saccharin with illness. Apart from ease of conditioning, another feature of taste aversions is that rats will associate a novel taste with illness despite CS-US delays of up to $12 \mathrm{~h}$ (Revusky \& Garcia, 1970). In this context, the present experiment investigated the effect of introducing various delays between saccharin consumption and the injection of the drug.

\section{Method}

Subjects. The subjects were 24 male hooded rats of the same age and strain as those employed in Experiment 1. Each animal was housed individually and all were maintained at $85 \%$ of their preexperimental weights, which ranged from 351 to $426 \mathrm{~g}$. Water was available at all times in their home cages.

Apparatus. Same as in Experiment 1.

Procedure. The procedure was exactly the same as for Groups SIP-E and SIP-C in Experiment 1, except that, on the conditioning day, the animals were divided into four groups of six subjects each and were injected with $\mathrm{LiCl} 30,60,120$, or $240 \mathrm{~min}$ after being exposed to saccharin in the experimental chamber.

\section{Results and Discussion}

Because the conditions of Experiment 2 were identical to those in Experiment 1, the data from Groups SIP-E and SIP-C were included in the present study, the former as a no-delay group and the latter as the control group.

Figure 2 shows the mean saccharin intake of each of the poisoned groups on the test day. An analysis of variance indicated that the delay between saccharin consumption and $\mathrm{LiCl}$ injection was a significant determinant of the subsequent aversion $[F(4,25)=$ 


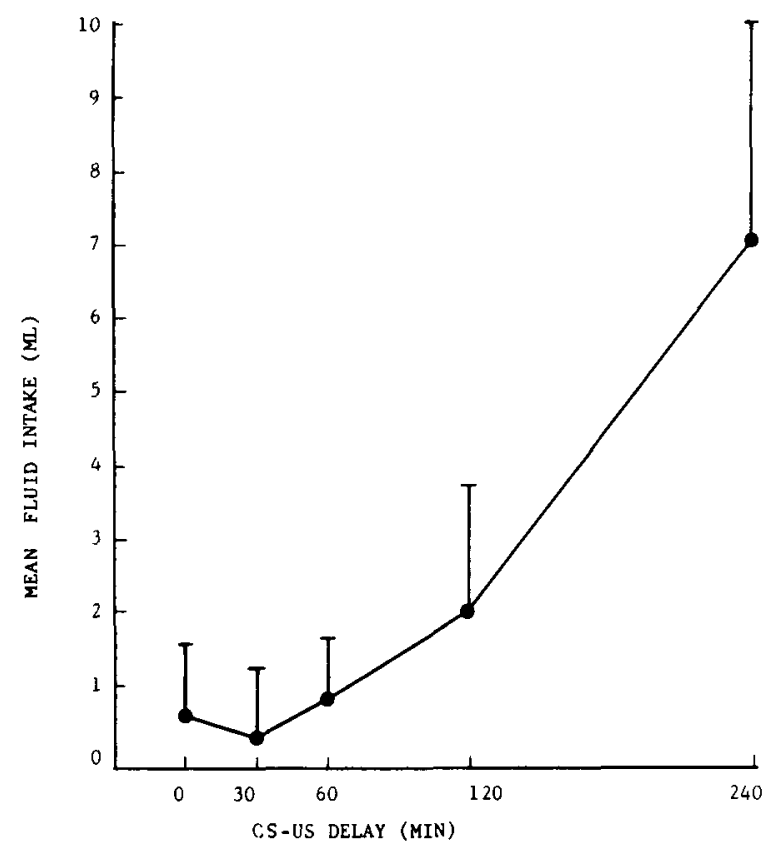

Figure 2. Mean saccharin intakes in the experimental chamber of each of the poisoned groups on the test day of Experiment 2. The data for the 0-min-delay group are from Experiment 1. The vertical lines indicate the standard deviations.

5.75, $\mathrm{p}<.01]$. Individual $\mathrm{t}$ tests revealed that the 240-min-delay group consumed significantly more saccharin than each of the remaining groups $[\mathrm{t}(10)>$ $3.17, \mathrm{p}<.01$, in all cases). No other comparisons were significant. Further, all of the poisoned groups, including the 240-min-delay group, consumed significantly less saccharin on the test day than the nonpoisoned control group $[\mathrm{t}(10)>2.23, \mathrm{p}<.05$, in all cases].

These results indicate that as the delay between schedule-induced saccharin consumption and poisoning is increased, the subsequent aversion to saccharin is attenuated. However, even when there is a delay of 240 min between saccharin consumption and poisoning, a significant aversion to saccharin is still evidenced. As such, the delay gradient of taste aversion learning obtained with schedule-induced fluid consumption is similar to that observed in thirstmotivated rats (cf. Revusky \& Garcia, 1970). In this respect, the findings of the present experiment illustrate the similarity of the factors which can control schedule-induced and thirst-motivated drinking (Bond, 1973).
The present results indicate that schedule-induced saccharin consumption is amenable to suppression in a taste aversion paradigm. Further, the gradient of delay obtained with schedule-induced drinking is similar to that observed in thirst-motivated rats. As such, schedule-induced polydipsia has been shown to be sensitive to a wide variety of suppression procedures (cf. Bond et al., 1973).

\section{REFERENCES}

Bond, N. W. Some comparisons of operant behavior and schedule-induced polydipsia. Unpublished $\mathrm{PhD}$ thesis, University of Nottingham, 1973.

Bond, N. W., Blackman, D. E., \& Scruton, P. E. Suppression of operant behavior and schedule-induced licking in rats. Journal of the Experimental Analysis of Behavior, 1973, 20. 375-383.

Bond, N., \& Di Giusto, E. Amount of solution drunk is a factor in the establishment of taste aversion. Animal Learning \& Behavior, 1975, 3, 81-84.

Cope, C. L. Sanger, D. J., \& Blackman, D. E. Intragastric water and the acquisition of schedule-induced drinking. Behavioral Biology, 1976, 17, 267-270.

FALK, J. L. Production of polydipsia in normal rats by an intermittent food schedule. Science, 1961, 133, 195-196.

FALK, J. L. Motivational properties of schedule-induced polydipsia. Journal of the Experimental Analysis of Behavior, 1966, 9, 37-41.

Kalat, J. W., \& Rozin, P. "Learned safety" as a mechanism in long-delay taste-aversion learning in rats. Journal of Comparative and Physiological Psychology, 1973, 83, 198-207.

Millenson, J. R. A programming language for on-line control of psychological experiments. Behavioral Science, 1971, 16, 248-256.

Revusky, S. H., \& GARCia, J. Learned associations over long delays. In G. H. Bower \& J. T. Spence (Eds.), The psychology of learning and motivation: Advances in research and theory (Vol. IV). New York: Academic Press, 1970. Pp. 1-84.

Roll, D., Schaeffer, R. W., \& Smith, J. C. Effects of a conditioned taste aversion on schedule-induced polydipsia. Psychonomic Science, 1969, 16, 39-41.

\section{NOTE}

1. During the 23.5-h period between the last baseline session (B) and the conditioning session (C), the polydipsic animals (Groups SIP-E and SIP-C) drank a mean of $13.7 \mathrm{ml}$ of water in their home cages.

(Received for publication September 15, 1977; revision accepted February 21, 1978.) 